\title{
Genetic Dissection of Resistance to Anthracnose and Powdery Mildew in Medicago truncatula
}

\author{
Carine Ameline-Torregrosa, ${ }^{1}$ Marc Cazaux, ${ }^{1}$ Dariush Danesh, ${ }^{2}$ Fabien Chardon, ${ }^{3}$ Steven B. Cannon, ${ }^{2}$ \\ Marie-Thérèse Esquerré-Tugayé, ${ }^{1}$ Bernard Dumas, ${ }^{1}$ Nevin D. Young, ${ }^{2}$ Deborah A. Samac, ${ }^{2,4}$ Thierry \\ Huguet, ${ }^{5}$ and Christophe Jacquet ${ }^{1}$ \\ ${ }^{1}$ UMR 5546 CNRS-UPS, Pôle de Biotechnologie Végétale 24 Chemin Borde Rouge, 34326 Castanet-Tolosan, France; \\ ${ }^{2}$ Department of Plant Pathology, University of Minnesota, 495 Borlaug Hall, St. Paul 55108, U.S.A.; ${ }^{3}$ INRA, Unité de nutrition \\ azotée des plantes, Versailles, 78026 France; ${ }^{4}$ United States Department of Agriculture-Agricultural Research Service, \\ Plant Science Research, St. Paul, MN 55108, U.S.A.; ${ }^{5}$ Laboratoire Symbiose et Pathologie des Plantes (SP2) Pôle de \\ Biotechnologie Végétale, 31326 Castanet-Tolosan, France
}

Submitted 12 July 2007. Accepted 4 October 2007.

\begin{abstract}
Medicago truncatula was used to characterize resistance to anthracnose and powdery mildew caused by Colletotrichum trifolii and Erysiphe pisi, respectively. Two isolates of $E$. pisi (Ep-p from pea and $E p-a$ from alfalfa) and two races of $C$. trifolii (races 1 and 2) were used in this study. The A17 genotype was resistant and displayed a hypersensitive response after inoculation with either pathogen, while lines F83005.5 and DZA315.16 were susceptible to anthracnose and powdery mildew, respectively. To identify the genetic determinants underlying resistance in A17, two $F 7$ recombinant inbred line (RIL) populations, LR4 (A17 $\times$ DZA315.16) and LR5 (A17 × F83005.5), were phenotyped with $E$. pisi isolates and $C$. trifolii races, respectively. Genetic analyses showed that $i)$ resistance to anthracnose is governed mainly by a single major locus to both races, named $C t 1$ and located on the upper part of chromosome 4; and ii) resistance to powdery mildew involves three distinct loci, Epp1 on chromosome 4 and Epal and Epa2 on chromosome 5. The use of a consensus genetic map for the two RIL populations revealed that Ct1 and Epp1, although located in the same genome region, were clearly distinct. In silico analysis in this region identified the presence of several clusters of nucleotide binding site leucine-rich repeat genes. Many of these genes have atypical resistance gene analog structures and display differential expression patterns in distinct stress-related cDNA libraries.
\end{abstract}

Additional keywords: biotrophic pathogen, disease, fungi, legumes.

C. Ameline-Torregrosa and M. Cazaux contributed equally to this work.

Corresponding author: C. Jacquet; Fax: + 33 (0) 5621935 02; E-mail: jacquet@scsv.ups-tlse.fr

Present address of C. Ameline-Torregrosa: LIPM, UMR 441-2594, CNRS-INRA, 31326 Castanet-Tolosan, France.

Present address of S. B. Cannon: USDA-ARS-Corn Insects and Crop Genetics Research, Iowa State University, 1575 Agronomy Building, Ames 50011, U.S.A.

*The $e$-Xtra logo stands for "electronic extra" and indicates that a supplementary table is published online.

This article is in the public domain and not copyrightable. It may be freely reprinted with customary crediting of the source. The American Phytopathological Society, 2008.
Plant diseases are a major limitation to crop production worldwide. Despite the economic need to find novel disease resistance $(R)$ genes in legumes, only a few $R$ genes have been cloned in this plant family (Ashfield et al. 2003; Gao et al. 2005). Major constraints to genetic improvement of crop legumes are the size and ploidy of genomes and a lack of appropriate mapping populations and chromosome walking tools, and slow or inefficient transformation methods for many species. To overcome the roadblocks in crop legume improvement, the alfalfa relative Medicago truncatula has been developed as a model plant to study legume biology (Barker et al. 1990; Cook 1999). Key attributes of $M$. truncatula include a diploid $(2 \mathrm{n}=2 \mathrm{x}=16)$ and relatively small genome (approximately $500 \mathrm{Mbp}$ ), autogamous fertilization, and a close phylogenetic relationships to major crop legumes (Zhu et al. 2005) such as pea (Pisum sativum) and alfalfa (M. sativa). A wealth of genomic tools and data have been developed, notably an ongoing whole-genome sequencing effort (Young et al. 2005) and the release of approximately 230,000 expressed sequence tags (ESTs) in public databases.

M. truncatula is a host for a number of economically important legume pathogens. Surveys of $M$. truncatula collections revealed large variation among accessions for resistance to Peronospora trifoliorum (Yaege and Stuteville 2000), Colletotrichum trifolii (O'Neill and Bauchan 2000), Erysiphe pisi (Yaege and Stuteville 2002), Phoma medicaginis (O'Neill et al. 2003), Aphanomyces euteiches (Vandemark and Grunwald 2004), and Uromyces striatus (Kemen et al. 2005). For each pathogen, accessions were identified with phenotypes ranging from highly susceptible to resistant, indicating that $M$. truncatula is a potentially rich source of genes for resistance to legume diseases. In order to exploit this natural variation, new genetic tools, including microsatellite markers (Mun et al. 2006), genetic maps (Thoquet et al. 2002), and recombinant inbred lines (RILs), were developed during the past few years ( $T$. Huguet, unpublished results). We used these tools to map genes in the $M$. truncatula genome that are associated with resistance to two major fungal diseases of crop legumes.

C. trifolii, the causal agent of alfalfa anthracnose (Elgin and Ostazeski 1985; Mackie et al. 2003), attacks seedlings, resulting in poor stand establishment along with defoliation and stem girdling on adult plants leading to severe yield losses. Three races $(1,2$, and 4$)$ have been identified and characterized according to the responses of reference alfalfa cultivars. In this host plant, resistance to race 1 and race 2 was found to be conferred by two 
single dominant genes, $A n_{l}$ and $A n_{2}$, respectively (Elgin and Ostazeski 1985). Race 4 was identified more recently in Australia and is able to overcome the resistance gene to race 2 but is controlled by $A n_{l}$ resistance (Mackie et al. 2003). No resistance gene to any Colletotrichum sp. has been cloned so far.

E. pisi is responsible for powdery mildew epidemics and causes significant yield losses in a number of legume crop species. In pea, severe infections can result in 25 to $35 \%$ yield reductions (Kumar and Singh 1981). Two recessive genes, $e r_{1}$ and $e r_{2}$, identified in some pea germplasm (Tiwari et al. 1997), confer resistance to E. pisi. Very recently, a single dominant resistance gene, named Er3, was detected in Pisum fulvum, a wild relative of cultivated pea (Fondevilla et al. 2007).

In previous work, three $M$. truncatula genotypes were selected in order to analyze resistance mechanisms against $C$. trifolii and E. pisi. A17-Jemalong is highly resistant to both pathogens, whereas F83005.5 is susceptible to $C$. trifolii race 1 (Torregrosa et al. 2004) and DZA315.16 is susceptible to a pea isolate of E. pisi (Foster-Hartnett et al. 2007). For each pathosystem, resistance was expressed as a localized hypersensitive response (HR) in which reactive oxygen species (ROS) and phenolics were generated. The two parental lines selected for each pathosystem were crossed. From the resulting progeny, two RIL populations named LR4 (A17 $\times$ DZA315.16) and LR5 (A17 × F83005.5) were produced and used for genetic dissection of resistance to two races of $C$. trifolii and two different isolates of $E$. pisi. Here, we describe the use of these populations to locate the underlying $\mathrm{R}$ loci and describe the $R$ gene-related sequences found at those loci.

\section{RESULTS}

\section{Resistance of A17 genotype}

to $C$. trifolii and $E$. pisi is associated with an HR.

Earlier studies of $M$. truncatula collections identified pairs of lines displaying susceptibility and resistance to $C$. trifolii race 1 (Torregrosa et al. 2004) and to an E. pisi pea (Ep-p) isolate (Torregrosa et al. 2004). In order to gain further insight into the genetic determinants of resistance, we challenged each selected pair of $M$. truncatula lines with two additional isolates: $C$. trifolii race 2 and an isolate of $E$. pisi purified from naturally infected alfalfa. The phenotypes observed upon inoculation as well as views of the host surfaces by scanning electron microscopy (SEM) are shown in Figure 1.

Inoculation of F83005.5 plants with $C$. trifolii race 2 resulted in severe disease symptoms. Infected leaves wilted and, afterward, became brown and dry (Fig. 1A). On stems, gray lesions with dark-brown borders appeared 1 week after inoculation. The lesions usually coalesced and girdled the stems, which inevitably collapsed. Altogether, 70 to $80 \%$ of inoculated F83005.5 plants died. In contrast, the growth of A17 plants was not altered by inoculation. Only small localized necrotic lesions, typical of HR, occasionally were observed on leaves. SEM showed that $C$. trifolii hyphal development was stopped by localized plant cell death following appressorium formation and penetration (Fig. 1B). In F83005.5, the leaf tissue was totally macerated 7 days post inoculation (dpi) and colonized by mycelium. Sporulating acervuli formed on plant surfaces, indicating completion of the pathogen life cycle and confirming F83005.5 as a susceptible host (Fig. 1C).

Following inoculation of DZA315.16 with the E. pisi alfalfa isolate $(E p-a)$, fine powdery white spots were observed after 5 to $6 \mathrm{dpi}$ on the upper side of inoculated leaves. Some of these pathogen colonies coalesced and covered the entire leaf surface 10 dpi (Fig. 1D). Susceptibility of DZA315.16 was moderate to $E p-a$, whereas this line was fully susceptible to $E p$ - $p$, most leaves being fully covered by mycelium (Foster-Hartnett et al. 2007). The inoculated A17 leaves remained symptomless, indicating that this line is highly resistant. These results were confirmed by SEM. On inoculated leaves of A17, fungal development was arrested following appressorium formation (Fig. 1E) whereas, on DZA315.16, the mycelium penetrated epidermal

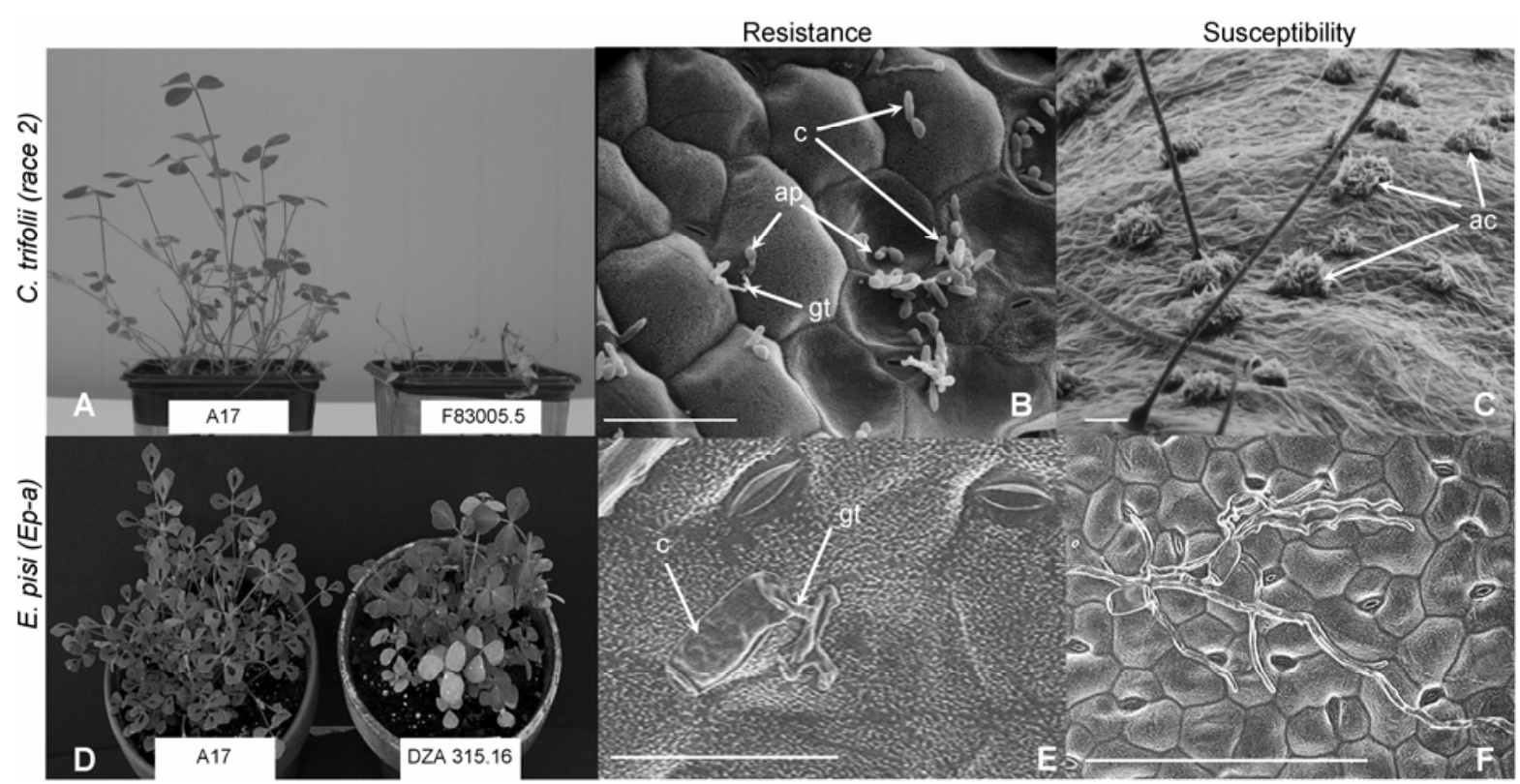

Fig. 1. Differential responses of parental lines to Colletotrichum trifolii race 2 and to the Erysiphe pisi alfalfa isolate (Ep-a). A through C, Parental lines (A17 and F83005.5) observed after C. trifolii race 2 inoculation. A, Whole plants observed at 15 days post inoculation (dpi). Most F83005.5 plants died whereas A17 plant growth remained unaffected. B, Scanning electronic microscopy (SEM) images of A17 leaves at 3 dpi. C, SEM of F83005.5 leaves at 5 dpi. Although collapsed cells undergoing a hypersensitive response (right bottom corner) are observed on A17 leaves, acervuli emerged on the surface of F83005.5. D through F, Reactions of A17 (resistant) and DZA315.16 (susceptible) upon inoculation of Ep-a. D, Whole plants at 10 dpi. Mycelia covered inoculated leaves of the susceptible host, whereas no growth was observed on comparable leaves of the resistant host. E, SEM showing a collapsed germinated E. pisi spore on resistant A17 leaf, 3 dpi. F, Hyphal development on susceptible DZA315.16, 3 dpi. Abbreviations: ac, acervuli; ap, appressorium; c, conidia; gt, germ tube. Bars represents $50 \mu \mathrm{m}$ in C, D, and E and $200 \mu \mathrm{m}$ in F. 
cells, colonized the leaf surface (Fig. 1F), and produced chains of conidia.

In summary, phenotyping parental lines with additional isolates of $C$. trifolii and $E$. pisi showed responses similar to those observed in previous studies (Foster-Hartnett et al. 2007; Torregrosa et al. 2004). In each pathosystem, A17 was highly resistant and able to halt pathogen development at a very early stage when the pathogen attempted to penetrate epidermal cells. Resistance mechanisms involved localized plant cell death (Fig. 1B and E) associated with accumulation of ROS and autofluorescent compounds (not shown), typical of incompatible HRs. The parental lines F83005.5 and DZA315.16 were susceptible to the new pathogen isolates $(C$. trifolii race 2 and $E p$ - $a$, respectively). Taken together with the previously characterized phenotypes, these differential responses allowed us to undertake a genetic analysis of resistance to $C$. trifolii and $E$. pisi by analyzing the RILs derived from crosses between the contrasting parental lines.

\section{Phenotyping of RIL populations.}

Two RIL populations named LR5 (A17 × F83005.5) and LR4 (A17 $\times$ DZA315.16) were phenotyped after C. trifolii and E. pisi inoculation, respectively.

Resistance to $C$. trifolii was assessed on detached leaves, as previously described (Torregrosa et al., 2004). In this previous report, intermediate phenotypes upon $C$. trifolii inoculation in the F2 population - in contrast to the clear-cut resistance or susceptibility observed for parental lines-led us to evaluate resistance to this fungus as a quantitative trait. Therefore, a disease score index (DSI) was created to classify disease symptoms observed on the $(\mathrm{A} 17 \times \mathrm{F} 83005.5) \mathrm{F} 2$ progeny inoculated with race 1 of $C$. trifolii. Inoculated leaves of resistant lines were recorded as class 1 or 2 while those of susceptible lines were scored as class 3 to 5 . Here, we used the same DSI (Fig. 2A) to screen 152 LR5 F7 or F8 RILs inoculated with C. trifolii race 1 and race 2 . The mean DSI obtained for parental lines inoculated with $C$. trifolii race 1 and race 2 were 1.78 and 1.91, respectively, for A17 and 4.71 and 4.82, respectively, for F83005.5. Upon phenotyping of the LR5 population, we identified a new class (class 6) (Fig. 2A), characterized by the rapid onset of coalesced lesions beneath the inoculated area. In the class 6 interaction, lesion formation occurred 3 dpi, compared with 5 or 6 dpi for leaves belonging to class 5 , and was followed immediately by severe, extensive chlorosis around the affected area. Wet macerated tissue, instead of the typical anthracnose dry lesions, generally was observed by 8 dpi. Plants with leaves belonging to class 6 were called "super-susceptible" lines. We identified two lines super-susceptible to each $C$. trifolii race and one additional line was found to be super-susceptible to both races. The distribution of DSI means
A

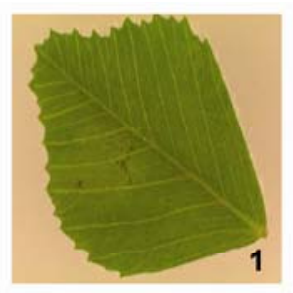

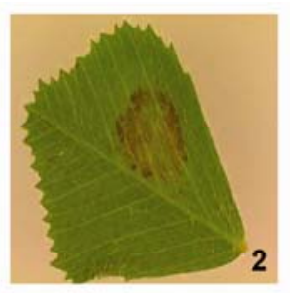

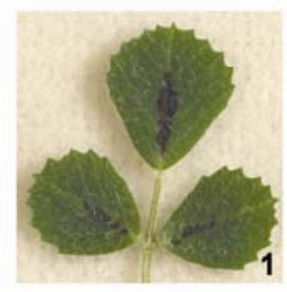

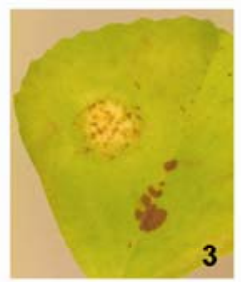
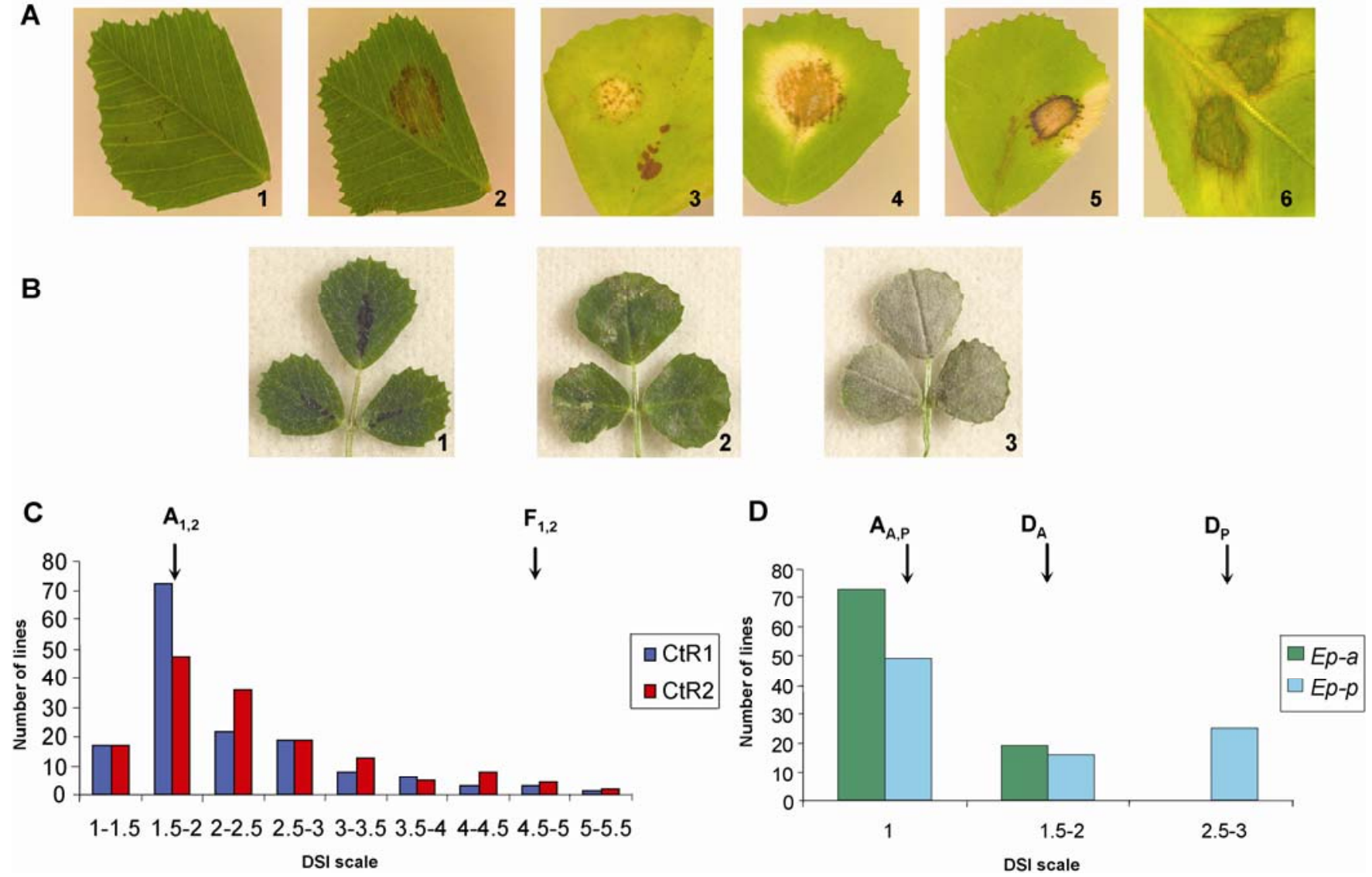

Fig. 2. Disease phenotypes following inoculation with Colletotrichum trifolii and Erysiphe pisi. A, Disease score index (DSI) established using detached leaflets of Medicago truncatula inoculated with $C$. trifolii at 8 days post inoculation (dpi). One drop of conidia was applied to each leaflet and symptoms were scored into six classes, where $1=$ no symptom or only a few isolated necrotic cells; $2=$ scattered necrotic cells; $3=$ chlorotic area beneath inoculation droplet; most of leaf green; 4 = severely chlorotic leaf and large necrotic lesions; $5=$ large necrotic area with dark-brown border, detection of acervuli, severe chlorosis, or both; and $6=$ large macerated areas that were observed as soon as 3 dpi beneath inoculum droplet, and severe chlorosis. Arrows indicate the points of inoculation. B, Disease index established on leaves of $M$. truncatula inoculated with E. pisi at 10 dpi; $1=$ complete resistance, no macroscopic symptoms; 2 = partial susceptibility, patches of mycelium were detected but did not coalesce; and $3=$ full susceptibility, trifoliate leaves were totally covered by mycelium. C, DSI means were first calculated with six to nine inoculated leaflets for each independent repeat. Final DSI values were obtained by calculating DSI means of three to five repeats for each recombinant inbred line. D, DSI means were calculated from scores recorded during two independent repeats. Arrows on $\mathrm{C}$ and D indicate the DSI of parental lines. $\mathrm{A}=\mathrm{A} 17 ; \mathrm{F}=\mathrm{F} 83005.5 ; \mathrm{D}=\mathrm{DZA} 315.16 ; 1$ or 2 indicates the DSI obtained following inoculation by $C$. trifolii race 1 or race 2, respectively; and A or P indicates the DSI following inoculation with Ep- $a$ or Ep-p, respectively. 
following inoculation with $C$. trifolii race 1 compared with $C$. trifolii race 2 in the LR5 population is presented in Figure 2C and shows a continuous monomodal distribution between class 1 (resistant) and class 5.5 (super-susceptible). Nevertheless, proportions of the different classes were different following inoculation with $C$. trifolii race 1 or race 2 . The percentage of RILs resistant to $C$. trifolii race 1 (mean score $<2.1$ ) was $62 \%$, whereas only $47 \%$ of RILs were resistant to C. trifolii race 2, indicating that this latter strain was more aggressive than race 1. Transgressive segregation was observed in both directions: several lines were more resistant than A17 while others were more susceptible than F83005.5 (Fig. 2C).

Reactions to E. pisi inoculation were rated on a scale of 1 to 3 (Fig. 2B) according to the extent of mycelium development on leaf surfaces of 92 F7 LR4 plants. Plants with class 1 phenotypes were considered to be resistant while plants with class 2 and 3 phenotypes were considered as partially or fully susceptible, respectively. A17 was scored as class 1 after inoculation with either E. pisi isolate, whereas mycelium developed and symptoms were observed on DZA315.16 plants. This latter line displayed a moderately (class 2 ) or highly susceptible phenotype (class 3) in response to $E p-a$ or $E p-p$, respectively. Distribution of the DSI (Fig. 2D) showed that $79 \%$ of the RILs were resistant to Ep-a and 54\% of RILs were resistant to Ep-p. No transgressive segregation was observed.

\section{Quantitative trait loci mapping.}

Analysis of variance (ANOVA) performed among inoculated RIL populations showed highly significant genetic variation for resistance to different strains of $C$. trifolii and $E$. pisi $(P<0.0001)$, indicating a genetic basis for the resistance observed in the RIL populations. Heritability $\left(h^{2}\right)$ was high for resistance to race 1 and race 2 of $C$. trifolii $(0.66$ and 0.65 , respectively) and even higher for resistance to $E p$ - $a$ and $E p-p$ (0.80 and 0.81 , respectively) (Table 1$)$. Two distinct genetic framework maps for the LR4 and LR5 populations based on microsatellite markers already have been used to map quantitative trait loci (QTL) for other traits (Julier et al. 2007; Vailleau et al. 2007). In order to compare the QTL positions calculated for the two types of pathogens in our study, a consensus genetic map for the LR4 and LR5 populations was developed. In all, 70 markers were assigned to the eight linkage groups (LG) of M. truncatula, with a total estimated size of $580 \mathrm{cM}$ and an average distance of approximately $8.5 \mathrm{cM}$ between markers (T. Huguet, unpublished results). The segregation data for the DSI scores and molecular markers calculated by composite interval mapping (CIM) was analyzed with PLABQTL software as described in Material and Methods. Biometrical parameters describing each detected QTL are shown in Table 1. Genetic analysis identified three QTL for resistance to E. pisi. These QTL were different between the two E. pisi isolates; one major
QTL (Epp 1) explaining approximately 66\% of the resistance to Ep-p was found on LG4 and two other major QTL (Epal and Epa2) explaining 26 and $23 \%$ of resistance to Ep- $a$ were identified on LG5. Results obtained for $C$. trifolii, showed that a major QTL of resistance located on LG4 was detected for race 1 and race 2 . The biometrical parameters (peak of maximum likelihood, left marker, and confidence interval) were the same for both $C$. trifolii races, indicating that the same locus, named $C t 1$, is involved in resistance to race 1 and 2 . A minor QTL (Ct2) for resistance to race 1, explaining less than $10 \%$ of the phenotypic variation, was detected at the bottom of LG6. Surprisingly, the resistance allele at this minor QTL was inherited from the susceptible parent F83005.5. These results were calculated with the consensus genetic map (i.e., the map developed with markers common to both LR4 and LR5 genetic maps). With LR4 and LR5 complete maps, the size of confidence intervals was reduced. For example, Eppl and $C t 1$ span 4 and $3 \mathrm{cM}$, respectively. However, data obtained with the consensus map alone only allowed comparisons of respective QTL locations to be made and showed that genetic resistance components to E. pisi and C. trifolii are clearly distinct.

\section{Combined genomic and genetic analyses of the QTL on chromosome 4 suggest resistance gene analogs as putative candidates.}

The A17 resistant parental line used in this study is currently being sequenced (Young et al. 2005). Taking advantage of the anchoring of our genetic markers to the physical map, we performed an in silico analysis of the 12.6-cM genomic region located between MTE23 and MTE24, the two flanking markers of the LG4 QTL. Surveying the predicted gene content of bacterial artificial chromosomes (BAC) within this interval might yield valuable information about putative gene candidates. MTE23 is genetically positioned at the top of LG4 and is indirectly linked to AC144503 (discussed below).

Because resistance to both pathogens was associated with an $\mathrm{HR}$, the presence of resistance gene analogs (RGA) located in the QTL was investigated. Most cloned $R$ genes in plants belong to the nucleotide binding site leucine-rich repeat (NBS-LRR) family and share conserved sequence domains, notably in the NBS region (McDowell and Woffenden 2003). Using these specific features, a previous study revealed that the upper portion of chromosome 4 contained numerous RGA, mostly organized in clusters (Zhu et al. 2002). In the present study, we focused our search for RGA within the genomic region between the BAC containing MTE23 and MTE24, using the recent genome assembly release Mt1.0 of $M$. truncatula genome sequence (Fig. 3) The analyzed region was spanned by 36 BAC organized in 12 contigs that were scanned using consensus sequences from the NBS-LRR gene family (Ameline-Torregrosa et al. in press). This analysis identified a total of 31 RGA sequences. After re-

Table 1. Biometrical parameters for regions of the Medicago truncatula genome associated with resistance to Erysiphe pisi and Colletotrichum trifolii calculated with a consensus framework genetic map between LR4 and LR5 populations ${ }^{\mathrm{a}}$

\begin{tabular}{|c|c|c|c|c|c|c|c|c|c|}
\hline Inoculated pathogen & Heritability $\left(h^{2}\right)$ & LG & Position & Left marker & Support interval (cM) & LOD & $R^{2}(\%)$ & Allele $^{b}$ & Name of QTLs \\
\hline \multicolumn{10}{|l|}{ E. pisi } \\
\hline Pea isolate $(E p-p)$ & 0.81 & 4 & 10 & Mtic 331 & $6-14$ & 21.33 & 66.4 & A & Eppl \\
\hline \multirow[t]{2}{*}{ Alfalfa isolate $(E p-a)$} & 0.80 & 5 & 38 & MTE 33 & $32-44$ & 5.92 & 26.2 & A & Epal \\
\hline & & 5 & 78 & MTE 37 & $70-88$ & 5.03 & 22.7 & A & Epa2 \\
\hline \multicolumn{10}{|l|}{ C. trifolii } \\
\hline \multirow[t]{2}{*}{ Race 1} & 0.66 & 4 & 2 & MTE 23 & $0-4$ & 12.59 & 33.4 & A & $C t 1$ \\
\hline & & 6 & 62 & MTE 42 & $50-66$ & 2.48 & 7.4 & $\mathrm{~F}$ & $\mathrm{Ct} 2$ \\
\hline Race 2 & 0.65 & 4 & 2 & MTE 23 & $0-4$ & 17.28 & 41 & A & $C t 1$ \\
\hline
\end{tabular}

${ }^{\text {a }} \mathrm{LG}=$ linkage group of $M$. truncatula; Position = location (given in centimorgans (cM) from the top of the LG) of the likelihood peak (maximum log of the likelihood ratio [LOD]); Left marker = name of the closest marker on the left of the likelihood peak; $R^{2}=$ proportion of the phenotypic variance explained by individual quantitative trait loci (QTLs).

${ }^{\mathrm{b}} \mathrm{A}$ or $\mathrm{F}$ indicates that the A17 or the F83005.5 allele increases the resistance, respectively. 
moving incomplete or mis-annotated sequences as well as duplicated sequences from the common regions of two overlapping BAC, a total of $25 \mathrm{RGA}$ was retained for further analysis. A combined genetic and genomic analysis of these RGA (Fig. 3C) showed the distribution and numbers of RGA sequences along the genomic region located between the two genetic borders. Detailed links between FPC contigs, BAC numbers, and predicted RGA locations are given in a supplementary table. These results indicate that this region is particularly rich in RGA and also revealed that the maximum likelihood peak detected for resistance to the two races of $C$. trifolii is located in a genomic area where RGA density is maximal. This segment of three BAC is located at $4.4 \mathrm{cM}$ from the top of chromosome 4 and contains nine RGA. Such a straightforward correlation between RGA content and the maximum likelihood peak detected for resistance to Ep-p could not be established.

Of the 25 RGA, 10 belong to the toll-interleukin receptor (TIR)-NBS-LRR (TNL) gene family (Table 2). A structural
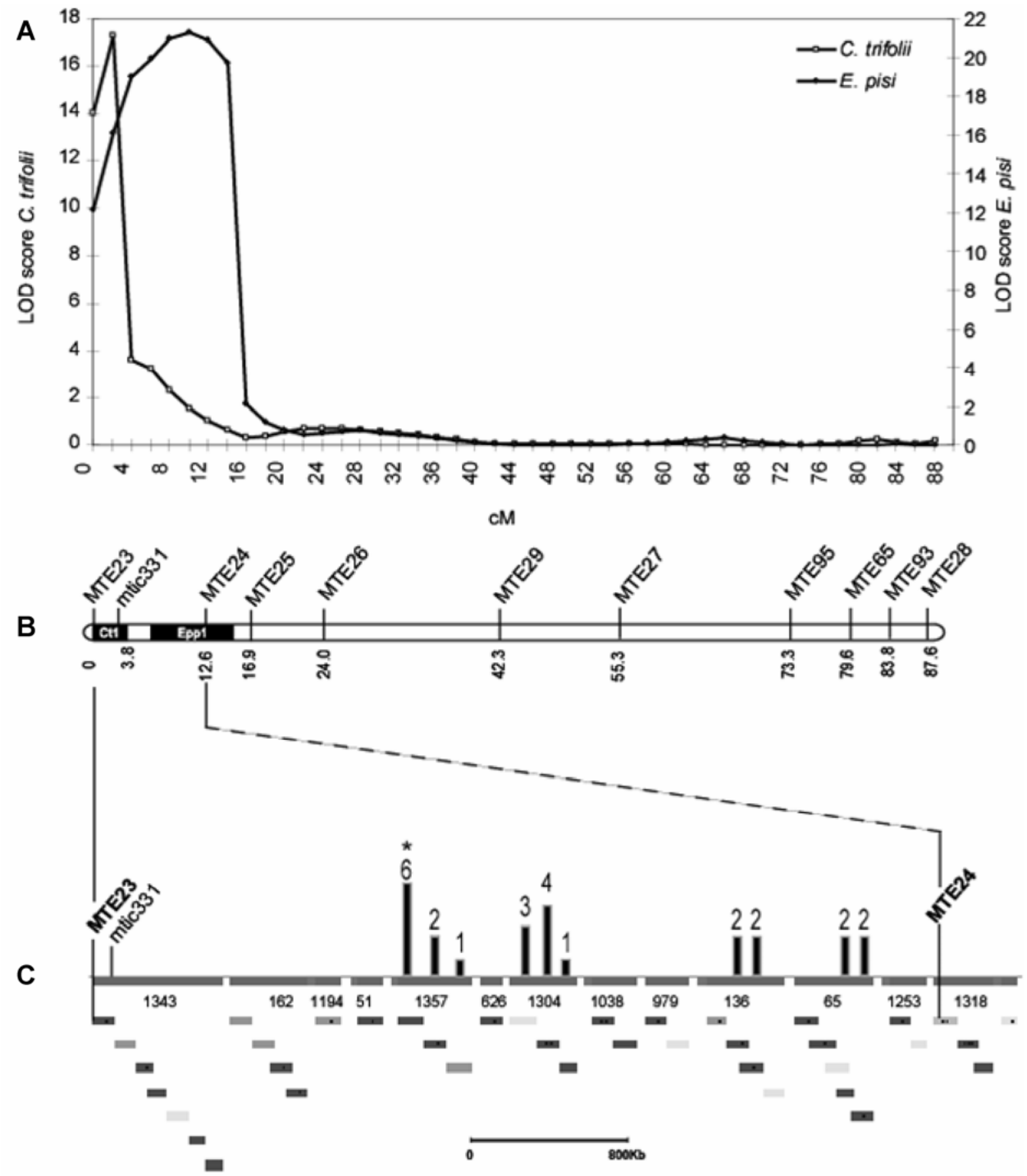

Fig. 3. Combined $C t 1$ and Eppl quantitative trait loci analyses with predictions of resistance gene analog (RGA) locations on the upper part of Medicago truncatula chromosome 4. A, Log of the likelihood ratio (LOD) distributions for resistance to Colletotrichum trifolii and to Erysiphe pisi on chromosome 4. B, Consensus genetic map of chromosome 4 with markers common to LR4 and LR5 maps. Relative genetic distances are indicated in centimorgans (cM) under each microsatellite marker used for both LR4 and LR5 genetic maps. With this consensus map, Ct1 and Eppl locations can be compared. C, Physical map of the Ct1 and Epp1 loci included between MTE23 and MTE24 markers revealed by the genetic analyses. Contig numbers and their BAC contents (visualized with the Genome Assembly Browser) are indicated, as well as the RGA positions and numbers detected through bioinformatic analysis. Location of Mtic 331 was shown on genetic and physical maps. This expressed sequence tag (EST) simple-sequence repeat marker allowed separation of $C t 1$ and Eppl in the genetic analyses. Star indicates the position of the only RGA expressed in the M. truncatula EST library isolated from leaves inoculated with $C$. trifolii. 
analysis of the other 15 RGA-like genes revealed atypical organization, such as a lack of the N-terminal domain preceding the NBS-LRR (NL) structure, additional TIR domains either before or after the classical TNL structure (TTNL and TNLT), or a repeat of the TIR-NBS domain upstream of the TNL coding region (TNTNL). Additional information on expression of these genes (Table 2) was provided by conducting a BLAST analysis of the $25 \mathrm{RGA}$ sequences against the $M$. truncatula EST database which consists of ESTs from 45 different cDNA libraries produced from plants under a wide range of physiological conditions. With the exception of one TTNL gene, ESTs were found for all identified RGA families. Of the 25 RGA, only one NL member was found to be expressed in the EST library derived from $C$. trifolii-inoculated leaves (DSIL EST library, GenBank number BF519144). This gene also was expressed in cell suspensions after fungal elicitor treatment and belongs to one of the three BAC contigs identified at the maximal likelihood peak of resistance to C. trifolii (Fig. 3C). Analysis of the other RGA did not reveal any biotic stress-specific expression, except for the two TNLT genes which were detected only in EST libraries derived from elicited cells.

\section{DISCUSSION}

In this work, we showed that M. truncatula parents of two RIL populations displayed contrasting phenotypes upon inoculation with two important fungi of cultivated legumes: $E$. pisi and $C$. trifolii. Both pathogens attack aerial parts of $M$. truncatula, although their lifestyles are distinctly different: $E$. pisi is a biotrophic obligate parasite (Faloon and Viljanen-Rollinson 2001) whereas $C$. trifolii is a hemibiotrophic pathogen (Perfect et al. 1999). In the two pathosystems, the A17 genotype displays resistance to all pathogen isolates tested. The fact that this accession was used to produce both the LR4 and LR5 RIL populations and is also the target of genome sequencing provided us the opportunity not only to compare genetic and genomic components of resistance against two distinct types of pathogens but also to study race-specific response mechanisms in M. truncatula.

Genetic analysis identified major QTL (Eppl, Epal, and Epa2) to E. pisi isolates that were clearly distinct from each other, based on their location in the genome (Eppl was located on LG4 whereas Epal and Epa2 were on LG5), as well as on their contribution to resistance (more than $66 \%$ for Eppl and 26 and 23\% for Epal and Epa2, respectively). Phenotype distribution in the F7 RIL population is consistent with 1:1 and 3:1 proportions of resistant and susceptible plants against $E p-p$ and Ep- $a$ isolates, suggesting the involvement of one and two major resistance genes to the pea and alfalfa isolates, respectively. The discovery of three new putative E. pisi resistance genes confirms that $M$. truncatula constitutes a valuable reservoir of resistance genes for major diseases of crop legumes. Several loci controlling natural resistance to powdery mildew have been described in barley (Schulze-Lefert and Vogel 2000) and Arabidopsis (Adam and Somerville 1996). In barley, the complex Mla locus governs powdery mildew resistance, which follows the classic gene-for-gene pattern of interaction; whereas, in Arabidopsis, alleles of the neighboring RPW8.1 and RPW8.2 genes mediate broad-spectrum resistance (Xiao et al. 2001). The results obtained in M. truncatula showed that genetic components underlying resistance to $E$. pisi are isolate specific, although cytological observations showed that infections by both isolates were arrested at the same stage of the infection process (i.e., immediately after appressorium formation). At this level, the resistance mechanism in A17 (a postpenetration HR) to $E$. pisi resembles that conferred by $e r_{2}$ in pea and by Er3 in P. fulvum (Fondevilla et al. 2007). Neither of these genes has been mapped on the pea genome, preventing any comparison with location of our three resistance QTL in M. truncatula.

Genetic analysis of resistance to $C$. trifolii indicated that a major QTL at the top of LG4 is responsible for resistance to race 1 and race 2 . Interval size and maximum peak for this QTL were the same for both races. Although the QTL detected for each race cannot be separated, the fact that all combinations of phenotypes (resistant to race 1 and 2, susceptible to race 1 and 2 , resistant to race 1 but susceptible to race 2 , and susceptible to race 1 and resistant to race 2) can be identified among the plants within the RIL population indicates that there are two $R$ genes, one recognizing each race. The small percentage of plants susceptible to race 1 and resistant to race 2 (3\%) indicated that these $R$ genes probably are located close together. Recent genetic analyses performed in alfalfa indicated that several markers and QTL located on top of alfalfa LG4 also were associated with increased resistance to $C$. trifolii race 1 and race 2 (Irwin et al. 2006; Mackie et al. 2007). Therefore, it appears that some genetic components involved in resistance to anthracnose are shared by $M$. truncatula and alfalfa, probably indicating the presence of orthologous $R$ genes on chromosome 4 of the two species.

Although the genetic analysis highlighted the QTL on top of LG4, genetic determinants of resistance to anthracnose in $M$. truncatula are not restricted to this region. Genetic analysis showed that this QTL explained approximately $40 \%$ of the phenotypic variation upon inoculation with $C$. trifolii. This result implies that other genetic determinants likely are involved in resistance and may act as modulators of resistance. This latter possibility was confirmed by transgressive segregation for the level of resistance obtained in the RIL population, as well as by the discovery of super-susceptible lines that display increased

Table 2. Occurrence of resistance gene analog (RGA) sequences from chromosome 4 between markers MTE23 and MTE24 in the Medicago truncatula expressed sequence tag (EST) database ${ }^{\mathrm{a}}$

\begin{tabular}{|c|c|c|c|c|c|c|c|}
\hline $\begin{array}{l}\text { RGA structural } \\
\text { organization }^{\mathbf{b}}\end{array}$ & $\begin{array}{l}\text { RGAs detected } \\
\text { within the QTL } \\
\text { on LG4 }\end{array}$ & $\begin{array}{l}\text { RGAs with sequences } \\
\text { detected in EST } \\
\text { libraries }\end{array}$ & Development & Symbiosis & $\begin{array}{l}\text { Abiotic } \\
\text { stress }\end{array}$ & $\begin{array}{l}\text { Colletotrichum trifolii- } \\
\text { inoculated leaf }\end{array}$ & $\begin{array}{l}\text { Elicited } \\
\text { cells }\end{array}$ \\
\hline NL & 7 & 4 & ++ & ++++ & - & + & + \\
\hline TNL & 10 & 3 & + & + & + & - & + \\
\hline TNLT & 2 & 2 & - & - & - & - & ++++ \\
\hline TNTNL & 5 & 3 & +++ & +++ & ++ & - & +++++ \\
\hline TTNL & 1 & 0 & - & - & - & - & - \\
\hline
\end{tabular}

${ }^{a}$ Development groups EST libraries obtained from developing flower, stem tissues, or germinating seed. QTL = quantitative trait loci. Symbiosis group EST libraries were produced from roots in interaction with Sinorhizobium meliloti or Glomus versiforme. Abiotic stress group EST libraries were produced from drought plantlets and UV- and $\gamma$-irradiated seedlings. Elicited cells group EST libraries were generated from cells treated with yeast cell wall extracts, $\beta$ glucan, or methyl jasmonate. Number of + indicates the number of hits between predicted cDNA sequences of an RGA and EST sequences detected in the various EST libraries. One RGA may have hits in several EST libraries; - indicates that no hit was detected in the EST libraries.

${ }^{\mathrm{b}} \mathrm{T}, \mathrm{N}$, and $\mathrm{L}$ are the protein domains detected: $\mathrm{T}=$ toll-interleukin receptor, $\mathrm{N}=$ nucleotide binding site, and $\mathrm{L}=$ leucine-rich repeat. 
susceptibility compared with F83005.5 plants. This result might suggest that putative regulators negatively affect plant basal or innate defense responses. In contrast, the detection of a resistance QTL derived from the susceptible F83005.5 parent indicates that there are other genetic determinants in $M$. truncatula that could increase the level of resistance and that these determinants can also be found in susceptible lines.

To better understand the mechanisms that might be involved in resistance to the two fungi, an in silico analysis was undertaken to search for RGA within the genomic regions defined by $C t 1$ and Eppl QTL. The systematic search for NBS domains that are found in most $R$ genes (van Ooijen et al. 2007) associated with other TIR, coiled-coil, and LRR domains indicated that several clusters of RGA were found within the QTL region. Clusters of RGA have been described in many plant species, including Arabidopsis (Meyers et al. 1999), soybean (Bhattacharyya et al. 2005), and bean (Geffroy et al. 2000). In our study, RGA density in the region surveyed was quite high, as exemplified by the identification of six RGA in one BAC. Therefore, the high density of putative $R$ genes in this QTL may explain why this genome region is associated with a broad spectrum of resistance to multiple fungi and fungal races.

The bioinformatic analysis revealed that many of the RGA in this region have atypical structural organization. TIR or LRR domains were either missing or duplicated around the NBS domain and, in some cases, were repeated within other coding regions. Among the 366 RGA detected throughout the $M$. truncatula genome (Ameline-Torregrosa et al. in press), only the upper part of chromosome 4 contains such atypical RGA. Phylogenetic analyses revealed recent and highly active evolution of $M$. truncatula chromosome 4. (Ameline-Torregrosa et al. in press). However, these atypical RGA are not legumespecific because an exhaustive survey of RGA in Arabidopsis identified similar features (Meyers et al. 2003). In this study, we demonstrated that even if the RGA are clustered, they are differentially expressed in a wide range of EST libraries. Taken together, these observations strongly suggest that the RGA identified may be the result of several rounds of tandem duplications followed by subfunctionalization events. Positive and purifying selection in $M$. truncatula may have driven the divergence of $R$ gene family members, generating new $R$ gene specificities as well as pseudogenes, as previously demonstrated in other plant species (Tiffin and Moeller 2006).

ESTs for all but one of these atypical RGA were detected in various cDNA libraries obtained from RNAs extracted from $M$. truncatula plants grown under a range of physiological conditions, indicating that none of them can be considered pseudogenes. Members of some of these RGA families, such as the TNTNL group, had ESTs detected in multiple libraries compared with others, such as TNLT, which were specifically associated with biotic stress-related libraries (Table 2). One EST library (named DSIL) was of particular interest because it was produced from A17 plants infected with C. trifolii. However, only 1 RGA out of the 25 candidates was detected in this library. The surprisingly low number of hits in this EST library might result from the fact that it was generated at a stage (5 dpi) in which resistance had already occurred and $R$ gene transcripts may be in low abundance compared with other induced messengers.

In conclusion, the use of two recently developed $M$. truncatula RIL populations combined with a consensus genetic map allowed us to identify genetic components that underlie resistance to major pathogens of cultivated legumes. This study also illustrates the exploitation of this model legume as a reservoir of new $R$ genes. A further major outcome of the RILs is the possibility to use the residual heterozygosity in some advanced lines for generating near-isogenic lines (NILs), differing only in the regions where QTL were found, or new RILs with crossing-over events within the QTL region. The NILs should confirm the involvement of the identified genomic regions in resistance to the tested pathogens; while RILs rich in cross-over events can help to reduce the size of the QTL region and target $R$ gene candidates. Such lines currently are being generated from the LR4 and LR5 populations. They will constitute powerful new tools to not only clone the relevant resistance genes but also identify signal transduction pathways activated by such $R$ genes through transcript profiling, without any interference that might result from the genetic background of the accession.

\section{MATERIALS AND METHODS}

\section{Plant materials and growth conditions.}

Three M. truncatula Gaertn. genotypes (A17, F83005.5, and DZA315.16) were used as reference lines in the two pathosystems. A17 is derived from the cv. Jemalong, DZA315.16 originates from a wild Algerian population, and F83005.5 comes from the French cv. Salernes. Seed were provided by J. M. Prospéri (INRA SGAP Laboratory, Mauguio, France). Seed were scarified with concentrated anhydrous sulphuric acid for $5 \mathrm{~min}$, washed three times in sterile water, sterilized for $3 \mathrm{~min}$ in $3 \%$ sodium hypochlorite, and rinsed three times in sterile water. Seed were vernalized for 1 day at $4^{\circ} \mathrm{C}$ and then allowed to germinate on $1 \%$ agar plates for 3 days at $15^{\circ} \mathrm{C}$ in the dark. Germinating seed were transferred into soil and cultivated in a growth chamber at $22^{\circ} \mathrm{C}$ with a $16-\mathrm{h}$ photoperiod $(200$ $\mu \mathrm{mol} / \mathrm{m}^{2} / \mathrm{s}$ ) and $8 \mathrm{~h}$ of darkness at $20^{\circ} \mathrm{C}$.

\section{Inoculation procedures.}

C. trifolii Bain, race 1 and 2, as determined with alfalfa cvs. Arc, Saranac, and Saranac AR (Elgin and Ostazeski 1982), were provided by M. B. Dickman (University of Nebraska, Lincoln U.S.A.). Cultivation of mycelium and production of conidia were performed as previously described (Torregrosa et al. 2004). Spore concentration was adjusted to $10^{6}$ conidia/ml in $0.01 \%$ Tween 20 . For whole-plant inoculation, 2-week-old plants were sprayed with $2 \mathrm{ml}$ of the spore suspension/plant and then were grown in a growth chamber as described above except that, for 3 days following inoculation, the relative humidity was $100 \%$. The detached leaf assay to phenotype RILs was performed as described by Torregrosa and associates (2004). Briefly, leaflets of young trifoliate leaves were placed on wet filter paper inside petri dishes and inoculated with a 6$\mu \mathrm{l}$ droplet of the spore suspension.

Two cultures of $E$. pisi established from natural infection of pea and alfalfa plants were maintained on Perfection 8771 pea plants grown in isolation at $22^{\circ} \mathrm{C}$ with a 16 -h photoperiod and $8 \mathrm{~h}$ of darkness at $16^{\circ} \mathrm{C}$. Four-week-old M. truncatula plants with two to three fully expanded compound leaves were inoculated in a $180-\mathrm{cm}$ settling tower with approximately 300 to 500 spores $/ \mathrm{cm}^{2}$ of leaflet area. After $20 \mathrm{~min}$, plants were returned to the growth chamber.

\section{RIL populations and analysis of resistance.}

Two RIL populations, LR4 and LR5, were derived from a cross of A17 with DZA315.16 and A17 with F83005.5, respectively. For each $E$. pisi isolate, two independent inoculation experiments were conducted on a set of 92 F7 LR4 RILs plus the parents. Disease assessment was carried out 8 dpi. The reaction to powdery mildew was rated on a scale of 1 to 3 as described in Figure 1B.

A set of 152 LR5 RILs was developed as single-seed descendants of the F7 and F8 generations. Two independent inoculations with $C$. trifolii race 1 or race 2 were performed on 143 
F7 lines and three to five inoculations were performed on the 152 F8 lines. Symptom classes (Fig. 1A) were recorded 8 dpi on six inoculated leaflets. Means of the DSI for race 1 and race 2 were calculated for each independent experiment and a final DSI score was attributed to each RIL by calculating the mean of the five biological repeats.

\section{QTL analysis.}

Framework genetic maps based on microsatellite markers were developed for the two RIL populations. LR4 and LR5 maps have been used already to identify other QTL (Julier et al. 2007; Vailleau et al. 2007). In this work, a final set of 70 markers common to the two genetic maps was retained to establish a consensus genetic framework map in order to compare the relative positions of $C t 1$ and $E p-p l$.

Replicate and genotype effects were examined by ANOVA with the PROC GLM procedure of SAS (SAS Institute, Cary, NC, U.S.A.) software. Genetic variance was estimated by using the PROC VARCOMP procedure of the SAS software, in which the genetic effect was assumed to be random. Heritability was estimated as $h^{2}=\sigma^{2} g /\left(\sigma^{2} g+\left[\sigma^{2} e / r\right]\right)$, with $\sigma^{2} g$ being the genetic variance, $\sigma^{2}$ e the residual variance, and $r$ the number of replicates. QTL were detected by CIM (Jansen 1993; Zeng 1994) using the software PLABQTL V1.1 (Utz and Melchinger 1996) combining the different sets of experiments. A purely additive model was employed. Individual cofactor sets were selected via stepwise regression for each trait. Final selection was for the model that minimized Akaike's information (AIC), a measure of the goodness-of-fit of the regression model (Jansen 1993). Empirical threshold values for the log of the likelihood ratio (LOD) scores were determined by computing 10,000 permutations (Churchill and Doerge 1994), using the "permute" command of the PLABQTL software. Therefore, the critical LOD score to indicate QTL significance was 2.3. QTL positions were determined at the local maxima of the LOD-curve plot in the region under consideration. Confidence intervals were set as the map interval corresponding to a 1LOD decline on either side of the LOD peak. The proportion of phenotypic variance explained by a single QTL was obtained by the square of the partial correlation coefficient $\left(R^{2}\right)$. Estimates of the additive effects of the QTL were computed by fitting a model including all putative QTL for a given trait. Sequences of left markers indicated in Table 1 are as follows: MTE23 (L: GGGTTTTTGATCCAGATCTT; R: AAGGTGGT CATACGAGCTCC), Mtic331 (L: CCCTCTTCTACCTCCTT TCCA; R: GGAAGAGAAGATGGGGGTGT), MTE24 (L: CT TGGCAAAATGTCAACTCT; R: GGAAAGGGGTTAGGTG AGTA), MTE33 (L: GTTTTGTCAATTTTCGAAGG; R: TGG GATAAAATTACGACACA), MTE37 (L: TTTTTGTTAGTTT GATTTTAGGTG; R: GCTACAAAGTCTTCTTCCACA), and MTE42 (L: TCAGGTAGGTTCAAAATTCC; R: CTGCAAG CCTGCATTATAC).

\section{SEM.}

Leaf samples collected between 1 and 7 dpi were fixed in $2.5 \%$ glutaraldehyde in $0.05 \mathrm{M}$ sodium cacodylate buffer, $\mathrm{pH}$ 7.2 , for $12 \mathrm{~h}$ at room temperature and then dehydrated in an ethanol series $(20,40,60,80$, and $100 \%)$. They were subjected to critical point drying (CPD 750 EmScope), coated with goldpalladium, and observed with a Hitachi S450 scanning electron microscope (Hitachi, Ibraki, Japan).

\section{Identification of RGA.}

The Ctl and Eppl genomic region was localized between two molecular microsatellite markers, MTE23 and MTE24. MTE23 corresponds to a marker indirectly linked to the top of chromosome 4 . It has been designed previously from an ex- pressed sequence (TC106363) and belongs to the right BAC end sequence (BES) of mth2-85p10, for which the left BES hits the physically anchored BAC AC144503 (Mtic 331), forming a BAC end bridge. MTE24 corresponds to the right marker bordering the QTL and is located on AC127674. BAC names present within the interval AC144503 (MTE23) to AC127674 (MTE24) were extracted from the medicago.org website and their respective sequences from GenBank. Putative NBS-LRR sequences were identified within this genome region according the strategy described by Ameline-Torregrosa and associates (in press). Briefly, the 1.0 draft genome assembly generated by the Medicago Genome Sequencing Consortium was used, with gene predictions from the International Medicago Genome Annotation Group. Candidate genes containing NBS domains were identified using blastp similarity to the CNL and TNL consensus sequences from plant-extended NBS domains and were aligned to a Hidden Markov Model calculated from this large collection (Cannon et al. 2002, 2004). For each sequence, specific domains of the NBS (P-loop, A to D domains, and GLPL) were verified manually. From an initial dataset of 31 sequences, 25 were retained after verification. The correspondence between the genetic and physical map was estimated for each BAC containing one or more RGA through the use of anchored markers MTE23 and MTE24 and the Genome Assembly Browser provided by the medicago.org website.

\section{In silico expression of RGA.}

Specific sequences of the NBS domain for each RGA detected within the interval delimited by markers MTE23 and MTE24 were identified and blasted against the EST database available from the National Center for Biotechnology website. Database hits were classified according their biological function and the EST counted.

\section{ACKNOWLEDGMENTS}

We thank M. B. Dickman (University of Nebraska, Lincoln, U.S.A.) for providing $C$. trifolii race 1 and race 2 .

\section{LITERATURE CITED}

Adam, L., and Somerville, S. C. 1996. Genetic characterization of five powdery mildew disease resistance loci in Arabidopsis thaliana. Plant J. 9:341-356.

Ameline-Torregrosa, C., Wang, B.-B., O’Bleness, M., Deshpande, S., Zhu, H., Roe, B., Young, N. D., and Cannon, S. Identification and characterization of NBS-LRR genes in the model plant Medicago truncatula. Plant Physiol. In Press.

Ashfield, T., Bocian, A., Held, D., Henk, A. D., Marek, L. F., Danesh, D., Peñuela, S., Meksem, K., Lightfoot, D. A., Young, N. D., Shoemaker, R. C., and Innes, R. W. 2003. Genetic and physical localization of the soybean Rpg1-b disease resistance gene reveals a complex locus containing several tightly linked families of NBS-LRR genes. Mol. PlantMicrobe Interact. 16:817-826.

Barker, D. G., Blanchi, S., Blondon, F., Detté, Y., Duc, G., Flament, P., Gallusci, P., Génier, P., Guy, P., Muel, X., Tourneur, J., Dénarié, J., and Huguet, T. 1990. Medicago truncatula, a model plant for studying the molecular genetics of the Rhizobium-legume symbiosis. Plant Mol. Biol. Rep. 8:40-49.

Bhattacharyya, M. K., Narayanan, N. N., Gao, H., Santra, D. K., Salimath, S. S., Kasuga, T., Liu, Y., Espinosa, B., Ellison, L., Marek, L., Shoemaker, R., Gijzen, M., and Buzzell, R. I. 2005. Identification of a large cluster of coiled coil-nucleotide binding site-leucine rich repeattype genes from the Rps 1 region containing Phytophthora resistance genes in soybean. Theor. Appl. Genet. 111:75-86.

Cannon, S. B., Zhu, H., Baumgarten, A. M., Spangler, R., May, G., Cook, D. R., and Young, N. D. 2002. Diversity, distribution, and ancient taxonomic relationships within the TIR and non-TIR NBS-LRR resistance gene subfamilies. J. Mol. Evol. 54:548-562.

Cannon, S. B., Mitra, A., Baumgarten, A., Young, N. D., and May, G. 2004. The roles of segmental and tandem gene duplication in the evolution of large gene families in Arabidopsis thaliana. BMC Plant Biol. 4:10. 
Churchill, G. A., and Doerge, R. W. 1994. Empirical threshold values for quantitative trait mapping. Genetics 138:963-971.

Cook, D. R. 1999. Medicago truncatula: A model in the making! Curr. Opin. Plant Biol. 2:301-304.

Elgin, J. H., and Ostazeski, S. A. 1982. Evaluation of selected alfalfa cultivars and related Medicago species for resistance to race 1 and 2 anthracnose. Crop Sci. 22:39-42.

Elgin, J. H., and Ostazeski, S. A. 1985. Inheritance of resistance to race 1 and race 2 anthracnose in Arc and Saranac AR alfalfa. Crop Sci. 25:861-865.

Faloon, R., and Viljanen-Rollinson, S. 2001. Powdery mildew. Pages 2829 in: Compendium of Pea Diseases and Pests. J. M. Kraft and F. Plfleger, eds. American Phytopathological Society, St. Paul, MN, U.S.A.

Fondevilla, S., Torres, A. M., Moreno, M. T., and Rubiales, D. 2007. Identification of a new gene for resistance to powdery mildew in Pisum fulvum, a wild relative of pea. Breed. Sci. 57:181-184.

Foster-Hartnett, D., Danesh, D., Peñuela, S., Sharopova, N., Endre, G., VandenBosch, K., Young, N. D., and Samac, D. A. 2007. Molecular and cytological responses of Medicago truncatula to Erysiphe pisi. Mol. Plant Pathol. 8:307-319.

Gao, H., Narayanan, N. N., Ellison, L., and Bhattacharyya, M. K. 2005. Two classes of highly similar coiled coil-nucleotide binding-leucine rich repeat genes isolated from the Rps $1-k$ locus encode Phytophthora resistance in soybean. Mol. Plant-Microbe Interact. 18:1035-1045.

Geffroy, V., Sevignac, M., De Oliveira, J. C., Fouilloux, G., Skroch, P., Thoquet, P., Gepts, P., Langin, T., and Dron, M. 2000. Inheritance of partial resistance against Colletotrichum lindemuthianum in Phaseolus vulgaris and co-localization of quantitative trait loci with genes involved in specific resistance. Mol. Plant-Microbe Interact. 13:287-296.

Irwin, J. A. G., Aitken, K. S., Mackie, J., and Musial, J. 2006. Genetic improvement of lucerne for anthracnose (Colletotrichum trifolii) resistance. Australas. Plant Pathol. 35:573-579.

Jansen, R. C. 1993. Interval mapping of multiple quantitative trait loci. Genetics 135:205-211.

Julier, B., Huguet, T., Chardon, F., Ayadi, R., Pierre, J., Prosperi, J. M., Barre, P., and Huyghe, C. 2007. Identification of quantitative trait loci influencing aerial morphogenesis in the model legume Medicago truncatula. Theor. Appl. Genet. 114:1391-1406.

Kemen, E., Hahn, M., Mendgen, K., and Struck, C. 2005. Different resistance mechanisms of Medicago truncatula ecotypes against the rust fungus Uromyces striatus. Phytopathology 95:153-157.

Kumar, H., and Singh, R. B. 1981. Genetic analysis of adult plant resistance to powdery mildew in pea (Pisum sativum L.). Euphytica 30:147-151.

Mackie, J., Musial, J., O’Neill, N. R., and Irwin, J. A. G. 2003. Pathogenic specialisation within Colletotrichum trifolii in Australia, and lucerne cultivar reactions to all known Australian pathotypes. Aust. J. Agric. Res. 54:829-836.

Mackie, J., Musial, J. M., Armour, D. J., Phan, H. T., Ellwood, S. E., Aitken, K. S., and Irwin, J. A. 2007. Identification of QTL for reaction to three races of Colletotrichum trifolii and further analysis of inheritance of resistance in autotetraploid lucerne. Theor. Appl. Genet. 114:1417-1426.

McDowell, J. M., and Woffenden, B. J. 2003. Plant disease resistance genes: Recent insights and potential applications. Trends Biotechnol. 21:178-183.

Meyers, B. C., Dickerman, A. W., Michelmore, R. W., Sivaramakrishnan, S., Sobral, B. W., and Young, N. D. 1999. Plant disease resistance genes encode members of an ancient and diverse protein family within the nucleotide-binding superfamily. Plant J. 20:317-332.

Meyers, B. C., Kozik, A., Griego, A., Kuang, H., and Michelmore, R. W. 2003. Genome-wide analysis of NBS-LRR-encoding genes in Arabidopsis. Plant Cell 15:809-834.

Mun, J. H., Kim, D. J., Choi, H. K., Gish, J., Debelle, F., Mudge, J., Denny, R., Endre, G., Saurat, O., Dudez, A. M., Kiss, G. B., Roe, B., Young, N. D., and Cook, D. R. 2006. Distribution of microsatellites in the genome of Medicago truncatula: A resource of genetic markers that integrate genetic and physical maps. Genetics 172:2541-2555.
O'Neill, N. R., and Bauchan, G. R. 2000. Sources of resistance to anthracnose in the annual Medicago core collection. Plant Dis. 84:261-267.

O'Neill, N. R., Bauchan, G. R., and Samac, D. A. 2003. Reactions in the annual Medicago spp. core germ plasm collection to Phoma medicaginis. Plant Dis. 87:557-562.

Perfect, S. E., Hughes, H. B., O'Connell, R. J., and Green, J. R. 1999. Colletotrichum: A model genus for studies on pathology and fungal-plant interactions. Fungal Genet. Biol. 27:186-198.

Schulze-Lefert, P., and Vogel, J. 2000. Closing the ranks to attack by powdery mildew. Trends Plant Sci. 5:343-348.

Thoquet, P., Ghérardi, M., Journet, E.-P., Kereszt, A., Ané, J.-M., Prospéri, J.-M., and Huguet, T. 2002. The molecular genetic linkage map of the model legume Medicago truncatula: An essential tool for comparative legume genomics and the isolation of agronomically important genes. BMC Plant Biol. 2:1-13.

Tiffin, P., and Moeller, D. A. 2006. Molecular evolution of plant immune system genes. Trends Genet. 22:662-670.

Tiwari, K. R., Penner, G. A., and Warkentin, T. D. 1997. Inheritance of powdery mildew in pea. Can. J. Plant Sci. 77:307-935.

Torregrosa, C., Cluzet, S., Fournier, J., Huguet, T., Gamas, P., Prosperi, J. M., Esquerre-Tugaye, M. T., Dumas, B., and Jacquet, C. 2004. Cytological, genetic, and molecular analysis to characterize compatible and incompatible interactions between Medicago truncatula and Colletotrichum trifolii. Mol. Plant-Microbe Interact. 17:909-920.

Utz, H. F., and Melchinger, A. E. 1996. PLABQTL: A program for composite interval mapping of QTL. J. Agric. Genomics 2:1.

Vailleau, F., Sartorel, E., Jardinaud, M. F., Chardon, F., Genin, S., Huguet, T., Gentzbittel, L., and Petitprez, M. 2007. Characterization of the interaction between the bacterial wilt pathogen Ralstonia solanacearum and the model legume plant Medicago truncatula. Mol. Plant-Microbe Interact. 20:159-167.

Vandemark, G., and Grunwald, N. J. 2004. Reaction of Medicago truncatula to Aphanomyces euteiches race 2. Arch. Phytopathol. Plant Prot. 37:59-67.

van Ooijen, G., van den Burg, H. A., Cornelissen, B. J., and Takken, F. L. 2007. Structure and function of resistance proteins in solanaceous plants. Annu. Rev. Phytopathol. 45:43-72.

Xiao, S., Ellwood, S., Calis, O., Patrick, E., Li, T., Coleman, M., and Turner, J. G. 2001. Broad-spectrum mildew resistance in Arabidopsis thaliana mediated by RPW8. Science 291:118-120.

Yaege, J. R., and Stuteville, D. L. 2000. Reactions in the annual Medicago core germ plasm collection to two isolates of Peronospora trifoliorum from alfalfa. Plant Dis. 84:521-524.

Yaege, J. R., and Stuteville, D. L. 2002. Reactions of accessions in the annual Medicago core germ plasm collection to Erysiphe pisi. Plant Dis. 86:312-315.

Young, N. D., Cannon, S. B., Sato, S., Kim, D., Cook, D. R., Town, C. D., Roe, B. A., and Tabata, S. 2005. Sequencing the Genespaces of Medicago truncatula and Lotus japonicus. Plant Physiol. 137:1174-1181.

Zeng, Z. B. 1994. Precision mapping of quantitative trait loci. Genetics 136:1457-1468.

Zhu, H., Cannon, S., Young, N., and Cook, D. R. 2002. Phylogeny and genomic organization of the TIR and non-TIR NBS-LRR resistance gene family in Medicago truncatula. Mol. Plant-Microbe Interact. 15:529539.

Zhu, H., Choi, H.-K., Cook, D. R., and Shoemaker, R. C. 2005. Bridging model and crop legumes through comparative genomics. Plant Physiol. 137:1189-1196.

\section{AUTHOR-RECOMMENDED INTERNET RESOURCES}

The Medicago truncatula Sequencing Project, genome assembly release Mt1.0: www.medicago.org/genome

MENS-Medicago EST Navigation System: medicago.toulouse.inra.fr/Mt/EST

National Center for Biotechnology Information: www.ncbi.nlm.nih.gov 